\title{
On the non-homogeneous effect of financial transaction taxes
}

\author{
Patrick Thöni* \\ University of Copenhagen, Copenhagen, Denmark
}

Received: 31 January 2020

Revised: 17 July 2020

Accepted: 12 September 2020

\begin{abstract}
This paper investigates the impact of a financial transaction tax (FTT) in a classic financial market setting. The benchmark analysis is based on an extension of the model presented in Kyle (1985). Opposed to the existing literature, I am able to find equilibrium values with a linear tax. Results of the benchmark model confirm standard findings of FTT's, such as an increased bidask spread and an overall less deep market. Importantly, I find that the introduction of a tax leads to a non-linear pricing function. In turn, the model predicts a decrease in market depth and trading aggressiveness for small trades, whereas for larger trades the introduction of a FTT only leads to increased spreads and prices.
\end{abstract}

Keywords: FTT; financial microstructure; rational expectations JEL Classification Codes: D53, G14

\section{Introduction}

The introduction of a financial transaction tax has been a focal point of research in the financial field for over 50 years, with Keynes (1936) and Tobin (1978) as its earliest and possibly most notable proponents. Since its first inception, the idea behind a FTT was based on the belief that its introduction would help to correct some market imperfections by reducing the trading of a specific group of traders. Specifically, Keynes proposed a tax as a tool to discourage unproductive speculative trading, whereas Tobin's tax was more focused on its ability to reduce excess volatility in currency exchange rates. Both, though, were different expressions of the same idea, namely that markets are populated by too many noise traders, whose trades are not based on information and therefore create excess volatility. Stiglitz (1989) and Summers and Summers (1989) present slightly different arguments in favor of a transaction tax, based on the grounds that it would slow down excessive speculative trading. Naturally, opposing viewpoints have been provided by the literature, most notably by Scholes (1981), who argues rather for the distortionary and self-defeating nature of transaction taxes.

\footnotetext{
*E-mail: pt@econ.ku.dk
}

Citation: Thöni, P. (2020) On the non-homogeneous effect of financial transaction taxes, Economics and Business Letters, 9(3), 230-239.

DOI: 10.17811/ebl.9.3.2020.230-239 
Various studies have investigated the effect of transaction taxes on market liquidity, such as Dupont and Lee (2007), Subrahmanyam (1998), and Dow and Rahi (2000). In general, these studies are not able to draw an overall conclusion on the directional effect of a tax. Subrahmanyam (1998) finds that a tax can both increase or decrease market liquidity, depending on whether informed traders act competitively or in a monopolistic way. Additionally, he finds that a tax can have positive effects by incentivizing agents to acquire more long-term information than short-term information. Similarly, Dupont and Lee (2007) find that the effect of a tax can be both negative or positive, depending on the level of informational asymmetry in the market. Dow and Rahi (2000) study the effect of a tax on the profits of speculators and the risk-sharing opportunities for hedgers. Again, they find that the effect of the tax depends on informational parameters of the model.

The aim of this study is to help shed further light on the directional effect of a transaction tax while allowing for a more realistic tax design. The benchmark analysis is based on an extension of the market model introduced in Kyle (1985). That is, we have a market populated by three types of traders: a market maker, noise traders and informed traders who have perfect knowledge of the securities price before trading (insiders). We therefore have a classic microstructure model with asymmetric information. Effectively, this model resembles a rational expectation model of the type introduced in Grossmann (1976) and Grossmann and Stiglitz (1980), where agents act based on their expectations of the other agents behaviour.

Analysis of the benchmark model supports most of the previous findings of the effect of transaction taxes, that is, an overall increase in the bid ask spread as well as a decreased market depth and trading aggressiveness of the insider. Importantly, this study also finds an effect that differs from the existing literature. By modelling the transaction taxes in a linear fashion, we find that the market makers pricing function becomes non-linear. This, in turn, leads to a heterogenous effect of the tax across different order sizes, effectively rendering small trades more costly relative to large trades.

\section{Model}

As mentioned above, this model in many ways draws from the setting developed by Kyle (1985) which, together with the Glosten-Milgrom model, represents the benchmark of financial microstructure models in the presence of asymmetric information.

Setting and Notation. I assume a classic financial market setting with two assets, a risky security, $v=\mu+\epsilon$ with $\epsilon \sim N\left(0, \sigma_{\epsilon}^{2}\right)$ and a riskless bond whose interest rate is normalized to zero. The market is populated by three types of agents: a market maker, an informed investor and a liquidity/noise investor. The liquidity traders submit a random aggregate order $u$, with $u \sim N\left(0, \sigma_{u}^{2}\right)$. The informed investors on the other hand have advanced knowledge of the risky assets payoff and can make their market orders contingent on its value, $x=X(v)$. The other market participants see the value of the risky asset as a random variable with distribution $v \sim$ $N\left(\mu, \sigma_{\epsilon}^{2}\right)$. It is further assumed that only market orders are allowed. The market makers observe the net batched order $q=x+u$, from which they try to infer the value of $v$. Finally, a tax $t$ is introduced as a percentage cost of the value of every transaction.

The model outlined in this setting belongs to the family of rational expectation models, where agents form conjectures about each other's behaviour. In equilibrium, these conjectures then need to be satisfied. In this environment, the market makers know that the order flow will reflect information about the fundamental since some of the agents that trade in the market have advanced knowledge of the true value of $v$. In order to infer the value of the underlying from 
the order flow, they need to make a conjecture about the relationship of $x$ and $v$. I assume that this conjecture takes the following form:

$$
x=X(v))= \begin{cases}\beta(v-\mu(1+t)) & \text { if } v>\mu(1+t) \\ 0 & \text { if } \mu(1+t)>v>\mu(1-t) \\ \beta(v-\mu(1-t)) & \text { if } v<\mu(1-t),\end{cases}
$$

where $\beta$ represents the trading aggressiveness of the informed agent. The introduction of a tax implies that the order flow of the informed agent will follow a piecewise function, that is, there is a range of values of $v$ for which it is neither profitable to buy nor to sell ${ }^{1}$. The full order flow observed by the market maker is then given by:

$$
q= \begin{cases}\beta(v-\mu(1+t))+u & \text { if } v>\mu(1+t) \\ u & \text { if } \mu(1+t)>v>\mu(1-t) \\ \beta(v-\mu(1-t))+u & \text { if } v<\mu(1-t) .\end{cases}
$$

Market Clearance. Market makers are assumed to be competitive and risk-neutral. The clearance of the market is organized as follows. First, both informed and uninformed traders post their market orders, which are then batched together in a single net order, $q=x+u$. This order flow is observed by the market maker, which in turn will post a price at which he is willing to execute that order. Finally, the entire order will be routed to the market maker that offers the best price. This in turn means that the market makers engage in a Bertrand competition, that is, market makers will effectively post a price equal to the marginal cost. The market maker that posts the best price $p$ will get the order flow $q$ routed to him.

Trading Strategy. We now turn to the demand side of the market. The informed traders are assumed to b risk neutral. In this model, both the market maker as well as the informed trader execute strategies that depend on their assumption about each other's behaviour. In equilibrium, these assumptions need to be fulfilled. Therefore, the informed traders' optimal trading strategy will depend on the market makers' pricing policy. Intuitively, the deeper he expects the market to be, the more aggressive he can be in his trading strategy and vice versa. This effectively means that we are looking for a Nash equilibrium, where every agent behaves optimally given the other agents' behavior.

Given that the conjectured trading behavior of the informed trader is a piecewise linear function, it is reasonable to assume a similar functional form for the price. Therefore, I assume that the informed trader conjectures the following pricing function

$$
p= \begin{cases}\mu_{1}+\lambda_{1} q & \text { if } q(v)>b \\ \mu_{2}+\lambda_{2} q & \text { if } a<q(v)<b \\ \mu_{3}+\lambda_{3} q & \text { if } q(v)<a\end{cases}
$$

where $\lambda_{i}, \quad i=\{1,2,3\}$ can be interpreted as the level of illiquidity in the market for different trading sizes $^{2}$.

Equilibrium notion. The notion of equilibrium that is used for the economy above is the standard one for rational expectations. It is defined as a pair $x$ and $p$, such that the following two conditions hold:

- Profit Maximization: The trading strategies need to satisfy

$$
x(p, v) \quad \in \quad \max _{x(p, v)} \mathbb{E}[(v-p) x]
$$

\footnotetext{
${ }^{1}$ This is similar to the "no trade zone" found in Constantinides (1986). Effectively, the introduction of a tax creates a range of values of the underlying for which it is no longer profitable to trade.

${ }^{2}$ More precisely, as $\lambda$ increases, the impact of a trade on the price increases. Therefore, the higher $\lambda$, the less deep the market.
} 
- Market Efficiency: The pricing function of the market makers needs to satisfy

$$
p(x, v)=\mathbb{E}[v \mid q]
$$

In the setting above we only allow for a fixed size of the transaction tax, thus abstracting from a possible differential impact arising due to differences in tax rates. In general, for countries that have implemented ad valorem transaction taxes on equity of the type we consider, the tax rates are usually very low and vary mostly between 20 and 50 basis points (Matheson (2011). Such low tax rates are also advocated by the literature (Darvas and von Weizsäcker (2011), Pollin et al. (2002), Schmidt (2007)) mostly due to concerns that a high tax rate would overly impair liquidity and drive activity to different markets. In the context of our model, as will become clear in the next section, a higher tax rate would lead to an increased non-linearity of the pricing function, therefore simply increasing the adverse effect of the tax.

\section{Solution to equilibrium}

The introduction of a linear tax, as we saw in the previous section, creates non-linearities in the trading strategies of the informed traders and therefore in the pricing function of the market maker. Therefore, standard solution techniques for linear rational expectation models do not apply to this setting. In order to solve the model, I use a novel approach, based on a linear approximation. The solution to the model is defined in the following theorem.

Theorem 1. Under the market structure and equilibrium notion defined in the previous section, the equilibrium price set by the market maker is:

$$
p=\mathbb{E}[v \mid q]=\mu+\int_{\mu t}^{\infty} \epsilon f\left(\epsilon \mid q^{+}\right) d \epsilon+\int_{-\infty}^{-\mu t} \epsilon f\left(\epsilon \mid q^{-}\right) d \epsilon
$$

where

and

$$
f(\epsilon \mid q)=\frac{e^{-\frac{\left(\epsilon-\mu_{\epsilon \mid q}\right)^{2}}{2 \sigma_{\epsilon \mid q}^{2}}}}{\sqrt{2 \pi} \sigma_{\epsilon \mid q}}, \quad \mu_{\epsilon \mid q}=\frac{\beta \sigma_{\epsilon}^{2}(q+\beta \mu t)}{\beta^{2} \sigma_{\epsilon}^{2}+\sigma_{u}^{2}}, \quad \sigma_{\epsilon \mid q}=\sqrt{\left(1-\rho^{2}\right) \sigma_{\epsilon}^{2}}
$$

$$
q^{+}=\beta(\epsilon-\mu t), \quad q^{-}=\beta(\epsilon+\mu t) .
$$

The pricing function can be approximated by the following piecewise linear function:

$$
\mathbb{E}[v \mid q]=\mathbb{E}\left[v \mid q^{*}\right]+\left\{\begin{array}{lll}
c & \text { if } q(v)>b \\
d q & \text { if } a<q(v)<b \\
(-c) & \text { if } q(v)<a .
\end{array}\right.
$$

where $c, d$ are defined in the appendix. The optimal order placement for the informed trader is given by:

$$
X(v)= \begin{cases}\beta_{1}\left(v-\mu_{1}\right) & \text { if } q(v)>b \\ \beta_{2}\left(v-\mu_{2}\right) & \text { if } a<q(v)<b \\ \beta_{3}\left(v-\mu_{3}\right) & \text { if } q(v)<a\end{cases}
$$

where

$$
\beta_{1}=\frac{1}{2 \lambda_{1}} \quad, \quad \beta_{2}=\frac{1}{2 \lambda_{2}} \quad, \quad \beta_{3}=\frac{1}{2 \lambda_{3}} .
$$

The equilibrium can therefore be characterized by the pair $\beta_{i}, \lambda_{i}, i=\{1,2,3\}$, which is given by the solution to the following equations:

$$
\beta_{1,3}=\frac{1}{2 \lambda_{1,3}}, \quad \lambda_{1,3}=\frac{\beta_{1,3} \sigma_{\epsilon}^{2}}{\beta_{1,3}^{2} \sigma_{\epsilon}^{2}+\sigma_{u}^{2}}
$$


for $q(v)>b$ and $q(v)<a$, and

$$
\beta_{2}=\frac{1}{2 \lambda_{2}} \quad, \quad \lambda_{2}=\frac{\beta_{2} \sigma_{\epsilon}^{2}}{\beta_{2}^{2} \sigma_{\epsilon}^{2}+\sigma_{u}^{2}}+\left.\frac{\partial f(q)}{\partial q}\right|_{q=0}
$$

for $a<g(v)<b$.

For $q(v)>b$ and $q(v)<a$ we can solve the equations and obtain the following equilibrium values

$$
\lambda_{1,3}=\frac{\sigma_{\epsilon}}{2 \sigma_{u}}, \quad \beta_{1,3}=\frac{\sigma_{u}}{\sigma_{\epsilon}} .
$$

Note that these values do not depend on $t$, but only on $\sigma_{u}$ and $\sigma_{\epsilon}$. In fact, these are the same equilibrium values obtained when $t=0$. This result is intuitive if one considers the optimal pricing policy of the market makers. In fact, for high enough order flows, the pricing function has the same slope as the pricing function obtained in this model without taxation. Therefore, it follows that the equilibrium values for market depth and trading aggressiveness have to be the same as in the case without taxation. The way the tax impacts the market for high positive (negative) values of $q$, is by increasing (decreasing) the level of the price set by the market maker. Moreover, we have that increasing the variance of the noise trading decreases market depth and increases the informed trader's aggressiveness. The opposite is true for the variance of the asset. These results are intuitive, since increasing the variance of the noise trader effectively means increasing the noise of the order flow and therefore the informational content of the latter, which leads to a higher market depth. On the other hand, a higher variance of the fundamental asset price increases the informational advantage of the informed trader, which in turn increases the informational content of a given order flow and therefore decreases market depth.

The equilibrium $\beta_{2}$ and $\lambda_{2}$ are determined by the intersection of equations (8) in the $\lambda-\beta$ space. In Figure 1 we can see how the equilibrium is affected by the introduction of a tax. In fact, we have that the equilibrium, compared to a no tax environment, moves up and to the left of the original equilibrium, therefore decreasing market depth as well as the aggressiveness of the informed traders order placement. Moreover, as shown in Figure 2, the impact of $\sigma_{u}$ and $\sigma_{\epsilon}$ described above are retained for these equilibrium values.

Discussion. Therefore, we have that the introduction of a tax impacts market depth only for small order flows, while for larger trades the tax does not affect the market depth but only the average level of the price. This result is different to Subrahmanyam (1998) and Dow and Rahi (2000), which do not find a heterogeneous effect for different sizes of trades. Subrahmanyam also allows for an arbitrary amount of informed traders, which allows him to distinguish between the case of a monopolist informed trader and the case of multiple informed traders. Surprisingly, he finds that in the case of a monopolist informed trader, market liquidity actually increases with the introduction of a tax. On the other hand, the authors results for the case of multiple informed traders are very similar to the ones presented above, except that his model is not able to capture the non-linearity of the market makers response function. Dow and Rahi (2000) as well as Dupont and Lee (2007) also consider similar issues, but in the context of a Glosten and Milgrom (1985) type of model. While the former are interested in answering the question whether speculators are better or worse off with a tax, in Dupont and Lee (2007) the effects of the tax on spreads and depth are considered. Their main finding is that the effect of a tax depends on the level of information asymmetry in the market. My results are in line with theirs for the case of a high level of assymetry.

The intuition behind the results found in this paper is that a tax increases the uncertainty about the informational content of the order flow mainly for low levels of the latter. That is, compared to a market without taxation, small order flows have the potential to hide a larger informational 
Figure 1. Equilibrium for different tax levels

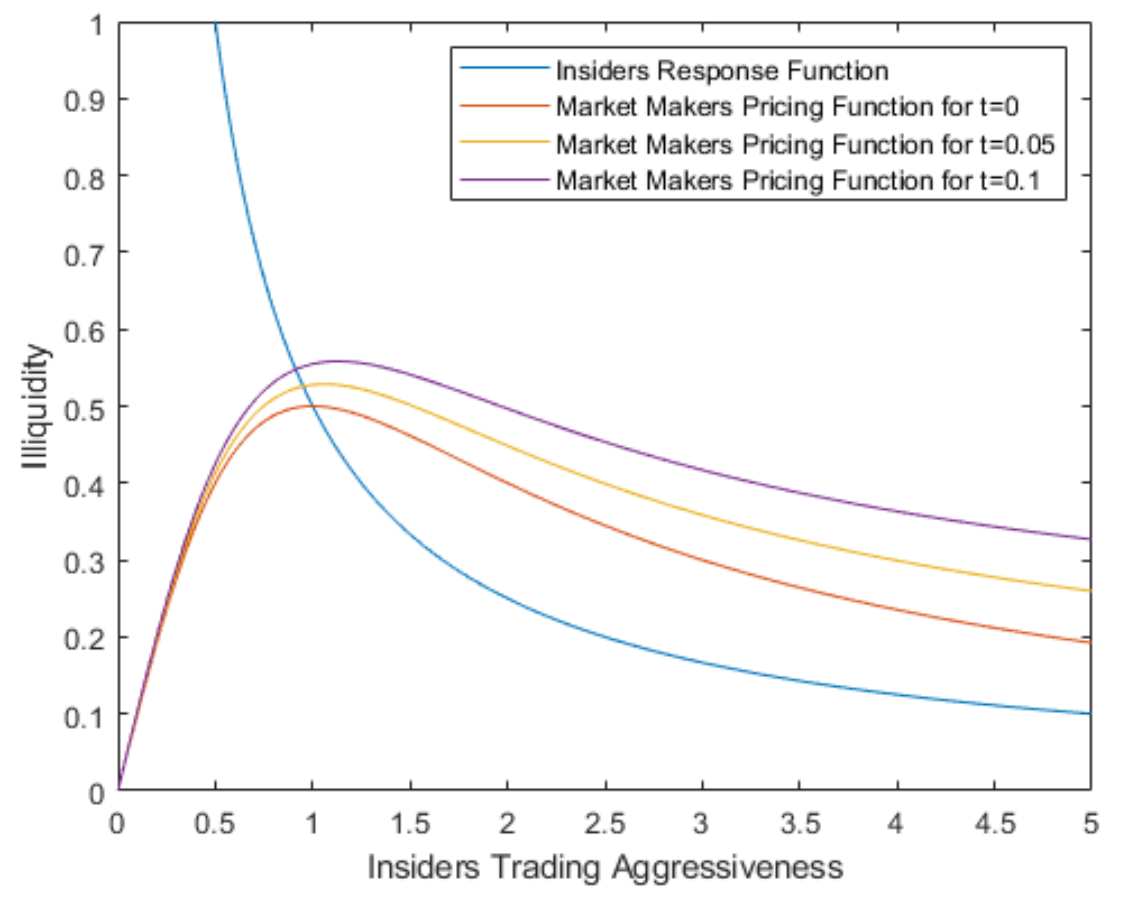

Note: The figure shows the equilibrium $\lambda$ and $\beta$ at different tax levels for $\mu=2, \sigma_{\epsilon}=1$ and $\sigma_{u}=1$.

Figure 2. Equilibrium for different $\boldsymbol{\sigma}_{\boldsymbol{\epsilon}}$ and $\boldsymbol{\sigma}_{\mathbf{u}}$.
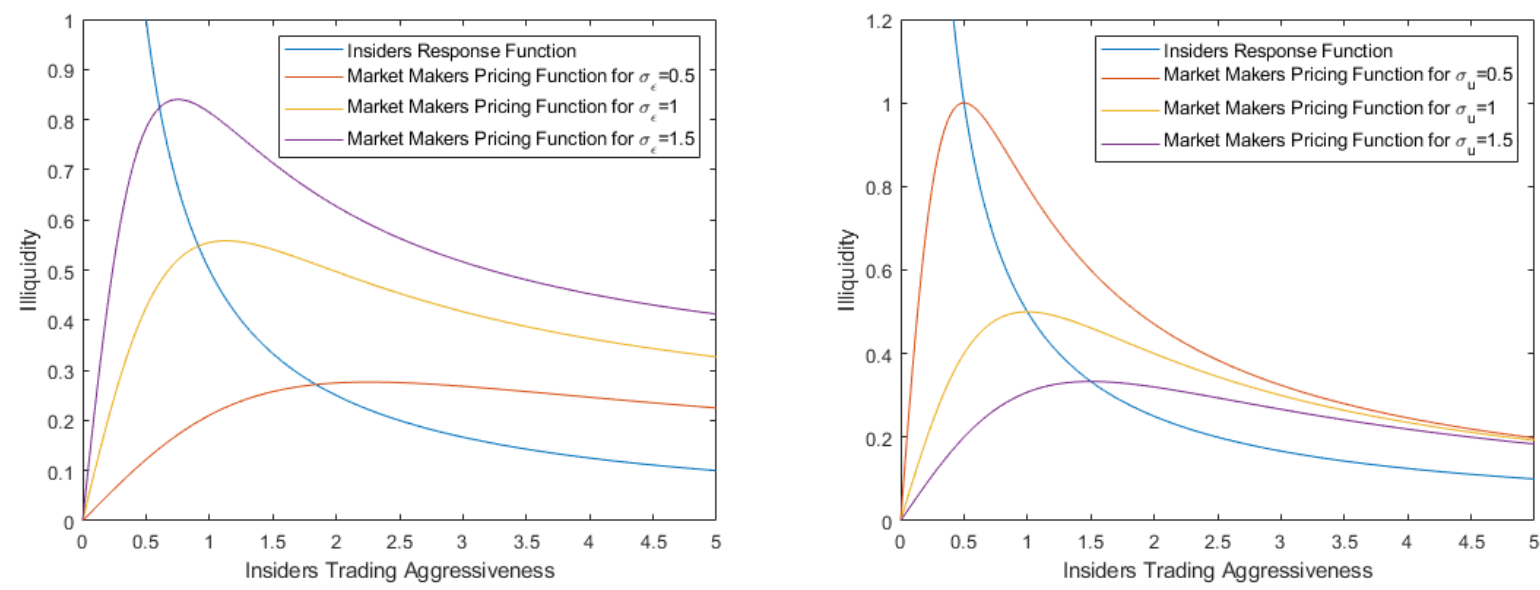

Note: The figure to the left shows the equilibrium $\lambda$ and $\beta$ for different volatilities of the asset and $\mu=2, \sigma_{\boldsymbol{u}}=1$. The figure to the right shows the equilibrium $\lambda$ and $\beta$ for different noise trading volatilities and $\mu=2, \sigma_{\epsilon}=2$.

advantage from the informed trader if a tax is introduced. For large order flows on the other hand, it is easier for the market maker to infer the true value of the asset, which leads to a deeper market, albeit with higher prices.

The analysis above abstracts from a few features that might be relevant in the broader discussion about the introduction of a transaction tax. Firstly, the paper focuses on the effect of the tax after its introduction, omitting possible effects of the period in between the announcement and the actual implementation of the tax. The modelling of such a period goes beyond the scope of the framework that we use in this paper, but it is worth discussing the possible effects of the announcement of a tax in light of the findings of this paper. If traders correctly anticipate that certain trades will get relatively more expensive after the introduction of a tax, they will try to execute those trades prior to the introduction. Depending on the 
aggressiveness and amount of such trading prior to the implementation of the tax, the market could experience increased volatilty as well as temporary arbitrage opportunities.

Additionally, we only consider the effect of the first implementation of a tax. In fact, one could expect a different behaviour if a tax had already been introduced previously in the market and traders are already familiar with such a regulatory framework. Generally, one would expect the incidence of the introduction of a tax in a market that had already been taxed before, or, alternatively, the incidence of a change of the design of a transaction tax to be lower compared to the effect we find in this paper. A deeper analysis of such issues is left for future research.

\section{Conclusion}

This study presents a rational expectation model in a classic setting with information asymmetry to study the effects of the introduction of a transaction tax. Different from previous studies, this paper introduces a linear tax to study its effects on the pricing behavior of the market makers. We are able to confirm most of the results found by previous studies, such as an increased bidask spread and a decreased market depth. Importantly, the model shows that the introduction of the tax leads the market makers to post non-linear pricing schedules with respect to the size of the order flow. In fact, the equilibrium market depth is lower for small orders, whereas the tax does not affect equilibrium values for large, positive or negative, orders. This analysis, therefore, suggests that the tax will have a larger effect on agents that post small orders, such as e.g. retail investors.

\section{References}

Constantinides, G. (1986) Capital Market Equilibrium with Transaction Costs, Journal of Political Economy 94(4), 842-62.

Darvas, Z., and von Weizsäcker, J. (2011) Financial Transaction Tax: Small is Beautiful, Society and Economy 33 , 449-473.

Dow, J., and Rahi, R. (2000) Should speculators be taxed?, The Journal of Business, 73(1), 89107.

Dupont, D. Y., and Lee, G. S. (2007) Effects of securities transaction taxes on depth and bidask spread, Economic Theory, 31(2), 393-400.

Glosten, L. R., and Milgrom, R. P. (1985) Bid, ask and transaction prices in a specialist market with heterogeneously informed traders, Journal of Financial Economics, 14, 70-100.

Grossman, S. J., and Stiglitz, J. E. (1980) On the Impossibility of Informationally Efficient Markets, The American Economic Review, 70(3), 393-408.

Grossman, S. J. (1976) On the efficiency of competitive stock markets when traders have diverse infformation, Journal of Finance, 31, 573-585.

Keynes, J. M. (1936) The General Theory of Employment, Interest and Money. London: Macmillan.

Kyle, A. S. (1985) Continuous Auctions and Insider Trading, Econometrica, 53(6), 1315-1335.

Matheson, T. (2011) Taxing financial transactions: issues and evidence, IMF working paper 54.

Pollin, R., Baker, D. and Schaberg, M. (2001) Securities Transaction Taxes for U.S. Financial Markets, Working Paper 20, Amherst: Political Economy Research Institute, University of Massachusetts.

Schmidt, R., (2007) The Currency Transaction Tax: Rate and Revenue Estimates (Ottawa: North-South Institute).

Scholes, M. S. (1981) The economics of hedging and spreading in futures markets, Journal of Futures Markets, 1(2), 265-286. 
Stiglitz, J. E. (1989) Markets, Market Failures, and Development, American Economic Review 79(2), 197-203.

Subrahmanyam, A. (1998) Transaction taxes and financial market equilibrium, Journal of Business 71, 81-118.

Summers, L. H., and Summers, V. P. (1989) When Financial Markets Work Too Well : A Cautious Case For A Securities Transactions Tax, Journal of Financial Services Research, 3, 261-286.

Tobin, J. (1978) A Proposal for International Monetary Reform, Eastern Economic Journal, 4(3-4), 153-159.

\section{Appendix - Proof of Theorem 1}

We start by proving equation 4 . We have the following:

$$
\begin{aligned}
\mathbb{E}[v \mid q]= & \mu+\mathbb{E}\left[\epsilon \mid[\beta(v-\mu(1+t))+u] 1_{\{v>\mu(1+t)\}}+[u] 1_{\{\mu(1-t)<v<\mu(1+t)\}}\right. \\
& \left.+[\beta(v-\mu(1-t))+u] 1_{\{v<\mu(1-t)\}}\right]
\end{aligned}
$$

This allows us to split up the conditonal expectation in the following way:

$$
\begin{aligned}
\mathbb{E}[v \mid q]= & \mu+\mathbb{E}[\epsilon \mid \beta(\epsilon-\mu t)+u, \epsilon>\mu t] \operatorname{Pr}[\epsilon>\mu t \mid q]+\mathbb{E}[\epsilon \mid u,-\mu t<\epsilon<\mu t] \\
& \operatorname{Pr}[-\mu t<\epsilon<\mu t \mid q]+\mathbb{E}[\epsilon \mid \beta(\epsilon+\mu t)+u, \epsilon<-\mu t] \operatorname{Pr}[\epsilon<-\mu t \mid q]
\end{aligned}
$$

which can be computed as

$$
\begin{gathered}
\mathbb{E}[\epsilon \mid \beta(\epsilon-\mu t)+u, \epsilon>\mu t] \operatorname{Pr}[\epsilon>\mu t \mid q]=\int_{-\infty}^{\infty} \epsilon f\left(\epsilon \mid q^{+}, \epsilon>\mu t\right) d \epsilon \operatorname{Pr}[\epsilon>\mu t \mid q], \\
\mathbb{E}[\epsilon \mid u,-\mu t<\epsilon<\mu t] \operatorname{Pr}[-\mu t<\epsilon<\mu t]=\mathbb{E}[\epsilon \mid-\mu t<\epsilon<\mu t] \operatorname{Pr}[-\mu t<\epsilon<\mu t]=0, \\
\mathbb{E}[\epsilon \mid \beta(\epsilon+\mu t)+u, \epsilon>\mu t] \operatorname{Pr}[\epsilon<-\mu t \mid q]=\int_{-\infty}^{\infty} \epsilon f\left(\epsilon \mid q^{-}, \epsilon<-\mu t\right) d \epsilon \operatorname{Pr}[\epsilon-\mu t \mid q],
\end{gathered}
$$

where $q^{+}$and $q^{-}$are respectively $\beta(\epsilon-\mu t)+u$ and $\beta(\epsilon+\mu t)+u$ and $f(\cdot)$ and $f(\cdot \mid \cdot)$ are the density and conditional density functions. Moreover the second expectation is 0 since $\epsilon$ and $u$ are independent and $\mathbb{E}[\epsilon \mid-\mu t<\epsilon<\mu t]$ is the mean of $\epsilon$ truncated symmetrically around its mean, 0 . After some algebra, the conditional expectation can be rewritten as

$$
\begin{aligned}
\mathbb{E}[v \mid q] & =\mu+\frac{f(\beta \epsilon-\beta \mu t+u)}{f(\beta \epsilon-\beta \mu t+u \mid \epsilon>\mu t) f(\epsilon>\mu t)} \int_{\mu t}^{\infty} \epsilon f(\epsilon \mid \beta \epsilon-\beta \mu t+u) d \epsilon \operatorname{Pr}[\epsilon>\mu t \mid q] \\
& +\frac{f(\beta \epsilon+\beta \mu t+u)}{f(\beta \epsilon+\beta \mu t+u \mid \epsilon>\mu t) f(\epsilon<-\mu t)} \int_{-\infty}^{-\mu t} \epsilon f(\epsilon \mid \beta \epsilon+\beta \mu t+u) d \epsilon \operatorname{Pr}[\epsilon<-\mu t \mid q] .
\end{aligned}
$$

Furthermore we have

and

$$
\begin{aligned}
\operatorname{Pr}[\epsilon>\mu t \mid q] & =\frac{\operatorname{Pr}[q \mid \epsilon>\mu t] \operatorname{Pr}[\epsilon>\mu t]}{\operatorname{Pr}[q]}=\frac{\operatorname{Pr}[\beta \epsilon-\beta \mu t+u \mid \epsilon>\mu t] \operatorname{Pr}[\epsilon>\mu t]}{\operatorname{Pr}[\beta \epsilon-\beta \mu t+u]} \\
& =\frac{f(\beta \epsilon-\beta \mu t+u \mid \epsilon>\mu t) f(\epsilon<-\mu t)}{f(\beta \epsilon-\beta \mu t+u)},
\end{aligned}
$$

$$
\begin{aligned}
\operatorname{Pr}[\epsilon<-\mu t \mid q \quad] & =\frac{\operatorname{Pr}[q \mid \epsilon<-\mu t] \operatorname{Pr}[\epsilon<-\mu t]}{\operatorname{Pr}[q]}=\frac{\operatorname{Pr}[\beta \epsilon+\beta \mu t+u \mid \epsilon<-\mu t] \operatorname{Pr}[\epsilon<-\mu t]}{\operatorname{Pr}[\beta \epsilon+\beta \mu t+u]} \\
& =\frac{f(\beta \epsilon+\beta \mu t+u \mid \epsilon>\mu t) f(\epsilon<-\mu t)}{f(\beta \epsilon+\beta \mu t+u)} .
\end{aligned}
$$

Therefore, $\mathbb{E}[v \mid q]$ becomes

$$
\mathbb{E}[v \mid q]=\mu+\int_{\mu t}^{\infty} \epsilon f(\epsilon \mid \beta \epsilon-\beta \mu t+u) d \epsilon+\int_{-\infty}^{-\mu t} \epsilon f(\epsilon \mid \beta \epsilon+\beta \mu t+u) d \epsilon .
$$

This conditional expectation can be rewritten as

$$
\mathbb{E}[v \mid q]=\mu+\int_{\mu t}^{\infty} \epsilon f(\epsilon \mid \beta \epsilon-\beta \mu t+u) d \epsilon+\int_{-\infty}^{-\mu t} \epsilon f(\epsilon \mid \beta \epsilon+\beta \mu t+u) d \epsilon=\mathbb{E}\left[v \mid q^{*}\right]+f(q),
$$

where $q^{*}=\beta(v-\mu)$, that is, the informed traders trading strategy when $t=0$ and $f(q)$ is a function with horizontal asymptotes for $q \rightarrow \infty$ and $q \rightarrow-\infty$. Therefore, the pricing function can be split up in a linear part, $\mathbb{E}\left[v \mid q^{*}\right]$, and a non-linear part, $f(q)$. The linear part is simply the conditional expectation given two jointly normally distributed variables $\left(v, q^{*}\right)$. This 
effectively means that for values of $q$ above and below a certain threshold, $\mathbb{E}[v \mid q] \approx \mathbb{E}\left[v \mid q^{*}\right] \pm$ $c=(\mu+c) \pm \lambda q^{3}$. For values in between those thresholds on the other hand, that is, for values of $q$ around 0 , we have $\mathbb{E}[v \mid q] \approx \mathbb{E}\left[v \mid q^{*}\right]+d q$. We can therefore approximate the pricing function by a piecewise linear function in the following way

$$
\mathbb{E}[v \mid q]= \begin{cases}\mathbb{E}\left[v \mid q^{*}\right]+c & \text { if } q(v)>b \\
\mathbb{E}\left[v \mid q^{*}\right]+d q & \text { if } a<q(v)<b=\mathbb{E}\left[v \mid q^{*}\right]+\left\{\begin{array}{lll}
c & \text { if } q(v)>b \\
d q & \text { if } a<q(v)<b \\
\mathbb{E}\left[v \mid q^{*}\right]-c & \text { if } q(v)<a .
\end{array} . \text { if } q(v)<a .\right.\end{cases}
$$

where $c=\lim _{q \rightarrow \infty} f(q), d=\left.\frac{\partial f(q)}{\partial q}\right|_{q=0}$ and the thresholds are given by $b=\frac{c}{d}$ and $a=\frac{-c}{d}$, where $c$ and $d$ are obtained in the following way. Since we have that $\mathbb{E}[v \mid q]=\mu+\int_{\mu t}^{\infty} \epsilon f(\epsilon \mid \beta \epsilon-\beta \mu t+u) d \epsilon+\int_{-\infty}^{-\mu t} \epsilon f(\epsilon \mid \beta \epsilon+\beta \mu t+u) d \epsilon=\mathbb{E}\left[v \mid q^{*}\right]+f(q)$, where $f(q)$ is

$$
\begin{aligned}
f(q)=-\frac{\beta \sigma_{\epsilon}^{2}(q+\beta \delta t) \operatorname{erf}\left(\frac{\mu t-\frac{\beta \sigma_{\epsilon}^{2}(q+\beta \mu t)}{\beta^{2} \sigma_{\epsilon}^{2}+\sigma_{u}^{2}}}{\sqrt{2} \sigma_{\epsilon \mid q}}\right)}{2\left(\beta^{2} \sigma_{\epsilon}^{2}+\sigma_{u}^{2}\right)}+\frac{\beta \sigma_{\epsilon}^{2}(q-\beta \mu t) \operatorname{erf}\left(\frac{-\frac{\beta \sigma_{\epsilon}^{2}(q-\beta \mu t)}{\beta^{2} \sigma_{\epsilon}^{2}+\sigma_{u}^{2}-\mu t}}{\sqrt{2} \sigma_{\epsilon \mid q}}\right)}{2\left(\beta^{2} \sigma_{\epsilon}^{2}+\sigma_{u}^{2}\right)} \\
+\frac{\sigma \epsilon \mid q \exp \left(-\frac{\left(\mu t-\frac{\beta \sigma_{\epsilon}^{2}(q+\beta \mu t)}{\beta^{2} \sigma_{\epsilon}^{2}+\sigma_{u}^{2}}\right)^{2}}{2 \sigma_{\epsilon \mid q}^{2}}\right)}{\sqrt{2 \pi}}-\frac{\sigma_{\epsilon \mid q} \exp \left(-\frac{\left(-\frac{\beta \sigma_{\epsilon}^{2}(q-\beta \mu t)}{\beta^{2} \sigma_{\epsilon}^{2}+\sigma_{u}^{2}}-\mu t\right)^{2}}{2 \sigma_{\epsilon \mid q}^{2}}\right)}{\sqrt{2 \pi}},
\end{aligned}
$$

and $\operatorname{erf}(\cdot)$ is the error function. We thus have

$$
\begin{aligned}
c=\lim _{q \rightarrow \infty} f(q)=\lim _{q \rightarrow \infty} & -\frac{\beta \sigma_{\epsilon}^{2}(q+\beta \delta t) \operatorname{erf}\left(\frac{\mu t-\frac{\beta \sigma_{\epsilon}^{2}(q+\beta \mu t)}{\beta^{2} \sigma_{\epsilon}^{2}+\sigma_{u}^{2}}}{\sqrt{2} \sigma_{\epsilon \mid q}}\right)}{2\left(\beta^{2} \sigma_{\epsilon}^{2}+\sigma_{u}^{2}\right)}+\frac{\beta \sigma_{\epsilon}^{2}(q-\beta \mu t) \operatorname{erf}\left(\frac{-\frac{\beta \sigma_{\epsilon}^{2}(q-\beta \mu t)}{\beta^{2} \sigma_{\epsilon}^{2}+\sigma_{u}^{2}}-\mu t}{\sqrt{2} \sigma_{\epsilon \mid q}}\right)}{2\left(\beta^{2} \sigma_{\epsilon}^{2}+\sigma_{u}^{2}\right)} \\
& +\frac{\sigma_{\epsilon \mid q} \exp \left(-\frac{\left(\mu t-\frac{\beta \sigma_{\epsilon}^{2}(q+\beta \mu t)}{\beta^{2} \sigma_{\epsilon}^{2}+\sigma_{u}^{2}}\right)^{2}}{2 \sigma_{\epsilon \mid q}}\right)}{\sigma_{\epsilon \mid q} \exp \left(-\frac{\left(-\frac{\beta \sigma_{\epsilon}^{2}(q-\beta \mu t)}{\beta^{2} \sigma_{\epsilon}^{2}+\sigma_{u}^{2}}-\mu t\right)^{2}}{2 \sigma_{\epsilon \mid q}^{2}}\right)} \\
& =\infty+\frac{\beta^{2} \sigma_{\epsilon}^{2} \mu t}{2\left(\beta^{2} \sigma_{\epsilon}^{2}+\sigma_{u}^{2}\right)}-\infty+\frac{\beta^{2} \sigma_{\epsilon}^{2} \mu t}{2\left(\beta^{2} \sigma_{\epsilon}^{2}+\sigma_{u}^{2}\right)}+0+0 \\
& =\frac{\beta^{2} \sigma_{\epsilon}^{2} \mu t}{\beta^{2} \sigma_{\epsilon}^{2}+\sigma_{u}^{2}} .
\end{aligned}
$$

Therefore, $c=\frac{\beta^{2} \sigma_{\epsilon}^{2} \mu t}{\beta^{2} \sigma_{\epsilon}^{2}+\sigma_{u}^{2}}$ is the horizontal asymptote of $f(q)$ as $q \rightarrow \infty$. For $q \rightarrow-\infty$, we have that the horizontal asymptote is $-c$.

Next, the slope of the middle part of the piecewise linear function is found by evaluating the derivative of $f(q)$ at 0 . We have

\footnotetext{
${ }^{3} \lambda$ here is the regression coefficient obtained when regressing $v$ on $q^{*}$, that is, $\frac{\operatorname{cov}\left(v, q^{*}\right)}{\operatorname{var}\left(q^{*}\right)}$
} 


$$
\begin{aligned}
d=\frac{\partial f(q)}{\partial q}= & -\frac{\beta \sigma_{\epsilon}^{2} \operatorname{erf}\left(\frac{\mu t-\frac{\beta \sigma_{\epsilon}^{2}(q+\beta \mu t)}{\beta^{2} \sigma_{\epsilon}^{2}+\sigma_{u}^{2}}}{\sqrt{2} \sigma_{\epsilon \mid q}}\right)}{2\left(\beta^{2} \sigma_{\epsilon}^{2}+\sigma_{u}^{2}\right)}+\frac{\beta \sigma_{\epsilon}^{2} \operatorname{erf}\left(\frac{-\frac{\beta \sigma_{\epsilon}^{2}(q-\beta \mu t)}{\beta^{2} \sigma_{\epsilon}^{2}+\sigma_{u}^{2}}-\mu t}{\sqrt{2} \sigma_{\epsilon \mid q}}\right)}{2\left(\beta^{2} \sigma_{\epsilon}^{2}+\sigma_{u}^{2}\right)} \\
& \left.+\frac{\beta^{2} \sigma_{\epsilon}^{4}(q+\beta \mu t) \exp \left(-\frac{\left(\mu t-\frac{\beta \sigma_{\epsilon}^{2}(q+\beta \mu t)}{\beta^{2} \sigma_{\epsilon}^{2} \sigma_{u}^{2}}\right)^{2}}{2 \sigma_{\epsilon \mid q}^{2}}\right)}{\sqrt{2 \pi} \sigma_{\epsilon \mid q}\left(\beta^{2} \sigma_{\epsilon}^{2}+\sigma_{u}^{2}\right)\left(\beta^{2} \sigma_{\epsilon}^{2}+\sigma_{u}^{2}\right)}-\frac{\beta^{2} \sigma_{\epsilon}^{4}(q-\beta \mu t) \exp \left(-\frac{\left(-\frac{\beta \sigma_{\epsilon}^{2}(q-\beta \mu t)}{\beta^{2} \sigma_{\epsilon}^{2}+\sigma_{u}^{2}-\delta t}\right)^{2}}{2 \sigma_{\epsilon \mid q}^{2}}\right)}{\sqrt{2 \pi} \sigma_{\epsilon \mid q}\left(\beta^{2} \sigma_{\epsilon}^{2}+\sigma_{u}^{2}\right)^{2}}\right) \\
& -\frac{\beta \sigma_{\epsilon}^{2}\left(-\frac{\beta \sigma_{\epsilon}^{2}(q-\beta \mu t)}{\beta^{2} \sigma_{\epsilon}^{2}+\sigma_{u}^{2}}-\mu t\right) \exp \left(-\frac{\left(-\frac{\beta \sigma_{\epsilon}^{2}(q-\beta \mu t)}{\beta^{2} \sigma_{\epsilon}^{2}+\sigma_{u}^{2}}-\mu t\right)^{2}}{2 \sigma_{\epsilon \mid q}^{2}}\right)}{\sqrt{2 \pi} \sigma_{\epsilon \mid q}\left(\beta^{2} \sigma_{\epsilon}^{2}+\sigma_{u}^{2}\right)} \\
& +\frac{\beta \sigma_{\epsilon}^{2}\left(\mu \mathrm{t}-\frac{\beta \sigma_{\epsilon}^{2}(q+\beta \mu t)}{\beta^{2} \sigma_{\epsilon}^{2}+\sigma_{u}^{2}}\right) \exp \left(-\frac{\left.\left(\mu \mathrm{t}-\frac{\beta \sigma_{\epsilon}^{2}(q+\beta \mu t)}{\beta^{2} \sigma_{\epsilon}^{2}+\sigma_{u}^{2}}\right)^{2}\right)}{2 \sigma_{\epsilon \mid q}^{2}}\right)}{\sqrt{2 \pi} \sigma_{\epsilon \mid q}\left(\beta^{2} \sigma_{\epsilon}^{2}+\sigma_{u}^{2}\right)}
\end{aligned}
$$

If we evaluate it at $q=0$ we obtain

$$
\begin{aligned}
& d=\left.\frac{\partial f(q)}{\partial q}\right|_{q=0}=-\frac{\beta \sigma_{\epsilon}^{2} \operatorname{erf}\left(\frac{\mu t-\frac{\beta^{2} \mu t \sigma_{\epsilon}^{2}}{\beta^{2} \sigma_{\epsilon}^{2}+\sigma_{u}^{2}}}{\sqrt{2} \sigma_{\epsilon \mid q}}\right)}{2\left(\beta^{2} \sigma_{\epsilon}^{2}+\sigma_{u}^{2}\right)}+\frac{\beta \sigma_{\epsilon}^{2} \operatorname{erf}\left(\frac{\frac{\beta^{2} \mu t \sigma_{\epsilon}^{2}}{\beta^{2} \sigma_{\epsilon}^{2}+\sigma_{u}^{2}}-\mu t}{\sqrt{2} \sigma_{\epsilon \mid q}}\right)}{2\left(\beta^{2} \sigma_{\epsilon}^{2}+\sigma_{u}^{2}\right)} \\
& +\frac{\beta \sigma_{\epsilon}^{2}\left(\mu \mathrm{t}-\frac{\beta^{2} \mu t \sigma_{\epsilon}^{2}}{\beta^{2} \sigma_{\epsilon}^{2}+\sigma_{u}^{2}}\right) \exp \left(-\frac{\left(\mu \mathrm{t}-\frac{\beta^{2} \mu t \sigma_{\epsilon}^{2}}{\beta^{2} \sigma_{\epsilon}^{2}+\sigma_{u}^{2}}\right)^{2}}{2 \sigma_{\epsilon \mid q}^{2}}\right)}{\sqrt{2 \pi} \sigma_{\epsilon \mid q}\left(\beta^{2} \sigma_{\epsilon}^{2}+\sigma_{u}^{2}\right)} \\
& -\frac{\beta \sigma_{\epsilon}^{2}\left(\frac{\beta^{2} \mu t \sigma_{\epsilon}^{2}}{\beta^{2} \sigma_{\epsilon}^{2}+\sigma_{u}^{2}}-\mu t\right) \exp \left(-\frac{\left(\frac{\beta^{2} \mu t \sigma_{\epsilon}^{2}}{\beta^{2} \sigma_{\epsilon}^{2}+\sigma_{u}^{2}}-\mu t\right)^{2}}{2 \sigma_{\epsilon \mid q}^{2}}\right)}{\sqrt{2 \pi} \sigma_{\epsilon \mid q}\left(\beta^{2} \sigma_{\epsilon}^{2}+\sigma_{u}^{2}\right)} \\
& +\frac{\beta^{3} \mu t \sigma_{\epsilon}^{4} \exp \left(-\frac{\left(\mu t-\frac{\beta^{2} \mu t \sigma_{\epsilon}^{2}}{\beta^{2} \sigma_{\epsilon}^{2}+\sigma_{u}^{2}}\right)^{2}}{2 \sigma_{\epsilon \mid q}^{2}}\right)}{\sqrt{2 \pi} \sigma_{\epsilon \mid q}\left(\beta^{2} \sigma_{\epsilon}^{2}+\sigma_{u}^{2}\right)^{2}}+\frac{\beta^{3} \mu t \sigma_{\epsilon}^{4} \exp \left(-\frac{\left(\frac{\beta^{2} \mu t \sigma_{\epsilon}^{2}}{\beta^{2} \sigma_{\epsilon}^{2}+\sigma_{u}^{2}}-\mu t\right)^{2}}{2 \sigma_{\epsilon \mid q}^{2}}\right)}{\sqrt{2 \pi} \sigma_{\epsilon \mid q}\left(\beta^{2} \sigma_{\epsilon}^{2}+\sigma_{u}^{2}\right)^{2}}
\end{aligned}
$$

Finally, $a$ and $b$, that is, the thresholds where the functions change, are simply found at the intersection of the three different linear functions. We therefore have $b=\frac{c}{d}$ and $a=\frac{-c}{d}$. 